\title{
Modelling and Analyzing Quadruped Robot Motion with Two Motors using Max-Plus Algebra
}

\author{
Laurensius I. Setiawan ${ }^{1, *}$ Laurent Simangunsong ${ }^{2,}$ Marcellinus A. Rudhito ${ }^{3}$
}

\author{
${ }^{1,2}$ Students of Mathematics Education Master Program, Universitas Sanata Dharma, Yogyakarta, Indonesia \\ ${ }^{3}$ Mathematics Education Master Program, Universitas Sanata Dharma, Yogyakarta, Indonesia \\ *Corresponding author. Email: setiawan.ian@gmail.com
}

\begin{abstract}
Nowadays mathematics has been widely used to program and control robots. One type of robots that exists is a robot with a type of propulsion in the form of legs, which is more effective for uneven and uphill terrain compared to the type of wheel drive. The study discusses quadruped robot motion with two motors using max-plus algebra. The study provides conclusions about the mechanically construct a four-legged robot with two motors, modeling the motion of a quadruped legged robot, and analyze of motion modeling of a quadruped legged robot.
\end{abstract}

Keyword: Quadruped robot, Max-Plus Algebra, eigenvectors

\section{INTRODUCTION}

Technology in modern times is developing very rapidly. Many uses of technology can help or facilitate human's work. One of the rapid technological developments is in the field of robotics. Robot is an electrical or electronic mechanical device that works automatically can work alone without outside control. Meanwhile, in a broad sense the robot means a system consisting of mechanical mechanisms that have an electrical control to carry out certain tasks [1]. At this time, the robot can not only make a sound but can also move [2]. Many examples of robots that can move such as the address finder using colours, flying robots, animalia robots, legged robots, and so on.

Nowadays mathematics has been widely used to program and control robots. One type of robot that is there is a robot with the type of propulsion in the form of legs, which is more effective for uneven and uphill terrain compared to the type of wheel drive type. Max-Plus algebra, in particular Switching Max-Plus Algebra has been used to model the legged locomotion. Research that has been done has modelled, optimized and controlled this legged robot by using Max-Plus algebra. The legged robot that is discussed is a quadruped robot, where the arms on the legs use a motor as movers. This robot moves based on the kinematics of motion applied to each leg arranged by servo motors [3]. Quadruped robot has a cycle of movement of footsteps that will continue to alternate, until the robot is ordered to work or stop. We develop the previous study [5] about switching max-plus model for legged locomotion using four motors. In the study, we use two motors to discuss the mechanical construction of a four-legged robot, modelling and analyzing the motion of the robot while walking using Max-Plus Algebra

\section{BASIC THEORY}

\subsection{Max-Plus Algebra [4]}

Max-Plus algebra is a set $\mathbb{R} \cup\{-\infty\}$ with $\mathbb{R}$ the set of all real numbers that are equipped with a maximum operation, denoted by $\oplus$ and the sum operation $\otimes$. Next $(\mathbb{R} \cup\{-\infty\}, \oplus, \otimes)$ denoted by $\mathbb{R}_{\text {max }}$ and $-\infty$ denoted with $\varepsilon$. The $\varepsilon$ element is a neutral element for operations $\bigoplus$ and 0 is the identity element for operations $\otimes$ [4]. A sum matriks MaxPlus $A, B \in \mathbb{R}_{\max }^{n \times m}$ defined by

$$
[A \oplus B]_{j i}=[A]_{j i} \oplus[B]_{j i}
$$

for $i=1,2, \ldots, m$ and $j=1,2, \ldots, n$

The matrix product $A \in \mathbb{R}_{\max }^{n \times 1}$ and $B \in \mathbb{R}_{\max }^{1 \times m}$ defined by

$$
\begin{aligned}
{[A \oplus B]_{j i} } & =\bigoplus_{k=1}^{l}\left([A]_{j i} \oplus[B]_{j i}\right) \\
& =\max \left\{[A]_{j i} \oplus[B]_{j i}\right\}
\end{aligned}
$$

for $i=1,2, \ldots, m$ and $j=1,2, \ldots, n$ 
Given $A \in \mathbb{R}_{\max }^{n \times n}$ with all circuits in $G(A)$ having non-positive weights, defined by

$A_{0}^{*}:=E \oplus A_{0} \oplus \ldots \oplus A_{0}^{\otimes^{n}} \oplus A_{0}^{\otimes^{n+1}} \oplus \ldots \quad$ and $A_{0}^{+}:=A_{0} \otimes A_{0}^{*}$, with $E$ is the identity matrix in Max-Plus algebra.

The max-plus linear (MPL) systems are linear in max-plus algebra. The MPL systems are the discrete event systems where the events are synchronized, there are no concurrent events and there are no choices to be made that determine the order of events. These systems are described by

$$
\begin{aligned}
x(k)= & A(k) \otimes x(k-1) \oplus B(k) \otimes \\
& u(k) \\
y(k)= & C(k) \otimes x(k)
\end{aligned}
$$

where $A \in \mathbb{R}_{\text {max }}^{n \times n}, B \in \mathbb{R}_{\max }^{n \times m}, C \in \mathbb{R}_{\max }^{p \times n}$, where $m$ is the number of inputs, $n$ is the number of states and $p$ is the number of outputs. The matrices $A, B, C$ often indicate the sum of maximization of transport times, or internal process times, etc. $x(k+1)$ indicates when the internal events happen for the $(k+1)^{t h}$ time, $u(k)$ indicates when the inputs became available for the $k^{t h}$ time and $y(k)$ indicates when the output events happen for the $(k)^{t h}$ time. In these systems the stade-vector is often the output. This means that $y(k)=x(k)$. Therefore, it is often left out of the equation and the rest is rewritten as

$$
\begin{aligned}
& x(k)=A(k) \otimes x(k-1) \oplus B(k) \otimes \\
& u(k)
\end{aligned}
$$

This form is called the explicit model as it only depends on the known values $x(k-1), u(k)$. The implicit form has the following format

$$
\begin{aligned}
x(k)= & A_{0}(k) \otimes x(k) \oplus A_{1}(k) \otimes \\
& x(k-1) \oplus B_{0}(k) \otimes u(k)
\end{aligned}
$$

If the matrices $A_{0}(k), A_{1}(k)$, and $B_{0}(k)$ are carefully constructed such that $A_{0}^{*}$ exists, which means $A_{0}$ is either nilpotent or has averages circuit mean of $e$ or less, than $A_{0}^{*} \otimes A_{1}=A, A_{0}^{*} B_{0}(k)=$ $B(k)$ and (5) can be rewritten into (4).

The switching of modes is controlled by a switching mechanism. This mechanism is represented by the switching variable $z(k)$ which may depend on the previous state-vectors $x(k-1)$, the previous mode $l(k-1)$, the input variable $u(k)$ and a control variable $v(k)$ :

$z(k)=\phi(x(k-1), l(k-$

$$
1), u(k), v(k)) \in \mathbb{R}_{\text {max }}^{n_{z}},
$$

where $\mathbb{R}_{\text {max }}^{n_{z}}$ is partitioned in $n_{m}$ subsets $Z^{(i)}, i=1$, $\ldots, n_{m}$. The set to which $z(k)$ belongs determines what mode the system will be in. In other words, if $z(k) \in Z^{(i)}$ then $1(k)=i$. The implicit model is described by

$$
x(k)=A_{0}^{l(k)}(k) \otimes x(k) \oplus A_{1}^{l(k)} \otimes x(k-
$$$$
\text { 1) } \oplus B_{0}^{l(k)}(k) \otimes u(k)
$$

The concepts of eigenvalues and eigenvectors about matrices in Max-Plus Algebra are given below

Definition 1 [4] The matrix $A \in \mathbb{R}_{\max }^{n \times n}$ is called irreducible if the weight graph is strongly connected.

Definition 2 [4] Let $A \in \mathbb{R}_{\max }^{n \times n}$. The scalar $\lambda \in \mathbb{R}_{\max }$ is called eigenvalue Max-Plus of matrix $A$ if there is a vector $v \in \mathbb{R}_{\max }^{n}$ where $v \neq \varepsilon_{n \times 1}$ that $A \otimes v=$ $\lambda \otimes v$. The vector $v$ is called eigenvector Max-Plus of matrices A that corresponding to $\lambda$.

Definition 3 [4] A SLMI (A, B, C, $x_{0}$ ) is called to be periodic with period $\lambda$, if $x(k)=\lambda^{\otimes^{k}} \otimes x(0)$, for $k=1,2,3, \ldots$

Definition 4 [4] The eigenvectors Max-Plus of matrix $A$ that corresponding to eigenvalue $\lambda_{\max }(A)$ called the fundamental Max-Plus eigenvector.

Theorem 1 [4] Let $A \in \mathbb{R}_{\max }^{n \times n}$. The scalar $\lambda_{\max }(A)$ is the maximum average of the elementary circuit in $G(A)$, and is the eigenvalue Max-Plus of matrix $A$.

Theorem 2 [4] Let $A \in \mathbb{R}_{\text {max }}^{n \times n}$. If the scalar $\lambda \in \mathbb{R}$ is the eigenvalue from Max-Plus algebra of matrix $A$, then $\lambda$ is the average weight of the circuit in $G(A)$.

Theorem 3 [4] If matrix $A \in \mathbb{R}_{\max }^{n \times n}$ irreducible, then A has an eigenvalue Max-Plus algebra.

\subsection{Basic Legged Robot Motion}

\subsubsection{Max-Plus Gait Scheduler [5]}

The first step taken to model the motion of a Quadruped robot is to determine the value of the variables for the transition event. Let $l_{i}(k)$ is the time when leg $i$ lift off and $t_{i}(k)$ is the time when the robot touchdown for $k$-th time iteration. For the leg $\mathbf{i}$ the time instance it touches down is equal to the time instance it lifted off the ground for the last time plus the time it stayed in flight, denoted by $\tau_{f}$

$$
t_{i}(k)=l_{i}(k)+\tau_{f}
$$

The time instance leg $i$ lifts off is equal to the time it touched down the last time plus the time it stayed in stance denoted by $\tau_{\mathrm{g}}$. This can then be written as

$$
l_{i}(k)=t_{i}(k-1)+\tau_{g}
$$

Suppose now our goal is to equate leg $i$ with leg $j$ so that leg $i$ is raised at $\tau_{\Delta}$ seconds after leg $\mathrm{j}$ has touched the ground. So we get a relationship 


$$
l_{i}(k)=\left[\begin{array}{ll}
\tau_{g} & \tau_{\Delta}
\end{array}\right] \otimes\left[\begin{array}{l}
t_{i}(k-1) \\
t_{j}(k-1)
\end{array}\right]
$$

\subsubsection{Modelling motion Quadruped robot}

Consider the relations written down in equation (8) and (9) and let the state vector for an $n$-legged robot be defined by

$x(k)=[\underbrace{t_{1}(k) \ldots t_{i}(k)}_{t(k)} \underbrace{l_{1}(k) \ldots l_{i}(k)}_{l(k)}]^{T}$

Based on equation (7) the term $B_{0}^{l(k)}(k) \otimes u(k)$ can be left out since there is no inputs $u(k)$. The system equations then take the form of

$$
\begin{aligned}
{\left[\begin{array}{l}
t(k) \\
l(k)
\end{array}\right] } & =\left[\begin{array}{c|c}
\varepsilon & \tau_{f} \otimes E \\
\hline \varepsilon & \varepsilon
\end{array}\right] \otimes\left[\begin{array}{l}
t(k) \\
l(k)
\end{array}\right] \\
& \oplus\left[\begin{array}{c|c}
E & \varepsilon \\
\hline \tau_{g} \otimes E & E
\end{array}\right] \otimes\left[\begin{array}{l}
t(k-1) \\
l(k-1)
\end{array}\right]
\end{aligned}
$$

Two $E$ matrices were added to the $A_{1}$ matrix which adds the requirement that the current touchdown event does not happen before the previous touchdown event and the current lift-off event does not happen before the previous lift-off event. Next the leg synchronization as described in equation (10) are added to this equation by adding the matrices $P$ and $Q$, which will be explained after this. The resulting implicit model is then defined as:

$$
\begin{aligned}
{\left[\begin{array}{l}
t(k) \\
l(k)
\end{array}\right] } & =\left[\begin{array}{c|c}
\varepsilon & \tau_{f} \otimes E \\
\hline P & \varepsilon
\end{array}\right] \otimes\left[\begin{array}{l}
t(k) \\
l(k)
\end{array}\right] \\
& \oplus\left[\begin{array}{c|c}
E & \varepsilon \\
\hline \tau_{g} \otimes E \oplus Q & E
\end{array}\right] \otimes\left[\begin{array}{l}
t(k-1) \\
l(k-1)
\end{array}\right]
\end{aligned}
$$

Model (11) can be rewritten as explicit model by using the solution of the the max-plus linear equation $x(k)=B \otimes x(k) \oplus c$ which is $x=$ $B^{*} \otimes c$. Substitute $B$ for $A_{0}$ and $c$ for $A_{1} \otimes$ $x(k-1)$, this results in

$$
\begin{aligned}
& x(k)=A_{0}^{*} \otimes A_{1} \otimes x(k-1) \\
& x(k)=A \otimes x(k-1)
\end{aligned}
$$

Where $A_{0}^{*} \otimes A_{1}$ is called the system matrix, $A_{0}^{*}$ has to exist in order to be able to solve this

$$
\begin{aligned}
& A_{0}=\left[\begin{array}{c|c}
\varepsilon & \tau_{f} \otimes E \\
\hline P & \varepsilon
\end{array}\right] \\
& A_{1}=\left[\begin{array}{c|c}
E & \varepsilon \\
\hline \tau_{f} \otimes E \oplus Q & E
\end{array}\right]
\end{aligned}
$$

So obtained

$$
A=A_{0}^{*} \otimes A_{1}
$$

Suppose $L_{1}, \ldots, L_{m}$ be sets of integers and let an $n$-legged system [6], such that

$$
\bigcup_{p=1}^{m} L_{p}=\{1, \ldots, n\}
$$

where $\forall i \neq j, l_{i} \cap l_{j}=\varnothing$

$L_{p}$ is considered to contain the indices of a set of legs that recirculates simultaneously. Define $r_{p}=$ $\# L_{p}$. A gait $\mathbf{G}$ is defined as an ordering relation of groups of legs:

$$
\boldsymbol{G}=L_{1}<L_{2}<\cdots<L_{m}
$$

\begin{tabular}{|c|c|c|c|c|c|c|c|c|}
\hline Leg & \multicolumn{4}{|c|}{ Matrix $P$} & \multicolumn{4}{|c|}{ Matrix $Q$} \\
\hline $\begin{array}{l}\{1,2\} \\
<\{3,4\}\end{array}$ & {$\left[\begin{array}{c}\varepsilon \\
\varepsilon \\
\tau_{\Delta} \\
\tau_{\Delta}\end{array}\right.$} & $\tau$ & & $\begin{array}{l}\varepsilon \\
\varepsilon \\
\varepsilon \\
\varepsilon\end{array}$ & {$\left[\begin{array}{l}\varepsilon \\
\varepsilon \\
\varepsilon \\
\varepsilon\end{array}\right.$} & $\begin{array}{l}\varepsilon \\
\varepsilon \\
\varepsilon \\
\varepsilon\end{array}$ & $\begin{array}{c}\tau_{\Delta} \\
\tau_{\Delta} \\
\varepsilon \\
\varepsilon\end{array}$ & $\left.\begin{array}{c}\tau_{\Delta} \\
\tau_{\Delta} \\
\varepsilon \\
\varepsilon\end{array}\right]$ \\
\hline $\begin{array}{l}\{1,3\} \\
<\{2,4\}\end{array}$ & {$\left[\begin{array}{c}\varepsilon \\
\tau_{\Delta} \\
\varepsilon \\
\tau_{\Delta}\end{array}\right.$} & $\begin{array}{l}\mathcal{E} \\
\mathcal{E} \\
\mathcal{E}\end{array}$ & & $\begin{array}{l}\varepsilon \\
\varepsilon \\
\varepsilon \\
\varepsilon\end{array}$ & {$\left[\begin{array}{l}\varepsilon \\
\varepsilon \\
\varepsilon \\
\varepsilon\end{array}\right.$} & $\begin{array}{c}\tau_{\Delta} \\
\varepsilon \\
\tau_{\Delta} \\
\varepsilon\end{array}$ & $\begin{array}{l}\varepsilon \\
\varepsilon \\
\varepsilon \\
\varepsilon\end{array}$ & $\left.\begin{array}{c}\tau_{\Delta} \\
\varepsilon \\
\tau_{\Delta} \\
\varepsilon\end{array}\right]$ \\
\hline $\begin{array}{l}\{1,4\} \\
<\{2,3\}\end{array}$ & {$\left[\begin{array}{c}\varepsilon \\
\tau_{\Delta} \\
\tau_{\Delta} \\
\varepsilon\end{array}\right.$} & $\varepsilon$ & $\begin{array}{l}\varepsilon \\
\varepsilon\end{array}$ & $\begin{array}{l}\varepsilon \\
\tau_{\Delta} \\
\tau_{\Delta} \\
\varepsilon\end{array}$ & {$\left[\begin{array}{l}\varepsilon \\
\varepsilon \\
\varepsilon \\
\varepsilon\end{array}\right.$} & $\begin{array}{c}\tau_{\Delta} \\
\varepsilon \\
\varepsilon \\
\tau_{\Delta}\end{array}$ & $\begin{array}{c}\tau_{\Delta} \\
\varepsilon \\
\varepsilon \\
\tau_{\Delta}\end{array}$ & $\left.\begin{array}{l}\varepsilon \\
\varepsilon \\
\varepsilon \\
\varepsilon\end{array}\right]$ \\
\hline
\end{tabular}

Based on [6], the matrices $P$ and $Q$ in equation (14) can be generated by:

$$
\begin{aligned}
& {[P]_{p q}=} \\
& \left\{\begin{array}{c}
\tau_{\Delta} \forall_{j} \in\{1, \ldots, m-1\} ; \forall_{p} \in l_{j+1} ; \forall_{q} \in l_{j} \\
\text { E otherwise }
\end{array}\right.
\end{aligned}
$$

$$
[Q]_{p q}=\left\{\begin{array}{c}
\tau_{\Delta} \forall_{j} \in l_{1} ; \forall_{q} \in l_{m} \\
\varepsilon \text { otherwise }
\end{array}\right.
$$

Next, the motion of footsteps $\boldsymbol{G}$ on a Quadruped robot consists of three possibilities of matrix $P$ and $Q$ is obtained in each step of the gait step, are:

Table 1. Matrix $P$ and $Q$ from each leg group

\section{RESULT AND DISCUSSION}

We develop quadruped legged robot with two motors based on the previous study [5]. We also use the Max-Plus Algebra concept regarding the quadruped legged robot [4,5]. We divide three parts to analyze the motion of quadrupped legged robot with two motors, as below. 


\subsection{Quadruped Robot Construction With Two Motor}

The robot that used in this study is the Lego Mindstorms EV3 robot. The Quadruped Robot used for simulation of leg movements is shown in Figure 1.

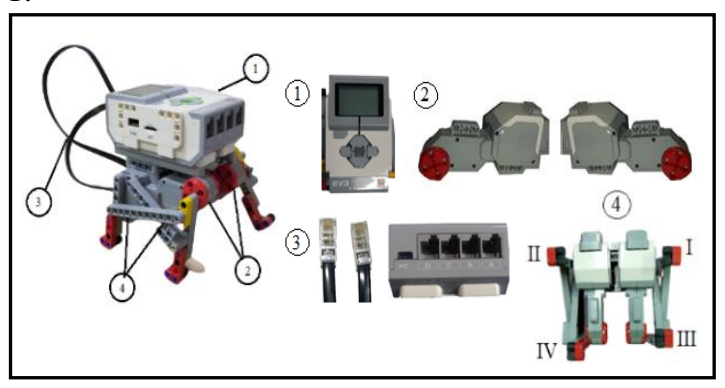

Figure 1 Quadruped Robot with two motors and its component

Description of Figure 1:

1. Robot Body Construction

Robot body construction is composed of Lego and other components.

2. EV3 Brick

EV3 Brick functions as a controller (as a brain and robot power source). Program compiled in Pyhton are then uploaded to EV3 Brick for compilation. EV3 Brick has processor to carry out different function together.

3. Two Motors

In the Lego Mindstorms EV3 robot, the motor used is the DC Servo motor which is equipped with an encoder that serves as feedback, so the control center can provide a current that matches the load on the motor. Two driving motors are programmed to move two legs while stepping together such as: $\{1,2\} \prec\{3,4\},\{1,4\} \prec\{2,3\}$ and $\{1,3\} \prec\{2,4\}$.

4. Connector

The connector serves to connect the EV3 Brick with two motors to drive the steps of the robot's legs. Two connectors are used and connected through two slots on EV3 Brick (slots B and C) and slots on both motors.

\subsection{Modelling Motion Quadruped Robot with Two Motors}

The following are the symbols used in the modelling of quadruped robot motion.
Table 2. The Symbols of modelling Motion Quadruped Robot

\begin{tabular}{|c|l|}
\hline $\begin{array}{c}\text { Symbol } \\
l_{i}(k)\end{array}$ & \multicolumn{1}{|c|}{ Definition } \\
\hline$t_{i}(k)$ & $\begin{array}{l}\text { Touch down time for leg } i \text { at iteration } \\
k\end{array}$ \\
\hline$\tau_{g}$ & $\begin{array}{l}\text { Time leg spends on the ground } \\
\text { (stance) }\end{array}$ \\
\hline$\tau_{f}$ & Time leg spends in flight (swing) \\
\hline$x(k)$ & $\begin{array}{l}\text { Full state vector of the touchdown and } \\
\text { lift off events }\end{array}$ \\
\hline$\tau_{\Delta}$ & Double time stance time \\
\hline
\end{tabular}

Based on the results of experiments, with slow motion, the values of parameters $\tau_{f}=1, \tau_{g}=2$, and $\tau_{\Delta}=1$. In the first part will be modeled when the movements of each foot start to move also in each iteration. For example, it is chosen for the foot group $\{1,2\} \prec\{3,4\}$, then using equations (8) and (9), modeling the time for each leg group shown in Table 3.

Table 3. Modelling the movement time of each leg

\begin{tabular}{|c|c|c|}
\hline $\begin{array}{l}\text { Leg } \\
\text { Group }\end{array}$ & $\begin{array}{c}\text { Time of Leg } \\
\text { Motion }\end{array}$ & Time Modelling \\
\hline \multirow[t]{2}{*}{$\begin{array}{l}\{1,2\} \\
\prec\{3,4\}\end{array}$} & $\begin{array}{l}t_{1}(k+1) \\
=t_{2}(k+1)\end{array}$ & $\begin{array}{l}=\max \left(t_{1}(k)+\tau_{g}\right. \\
+\tau_{f}, t_{3}(k)+\tau_{\Delta} \\
\left.+\tau_{f}\right) \\
=\max \left(t_{1}(k)+\tau_{g}\right. \\
+\tau_{f}, t_{4}(k)+\tau_{\Delta} \\
\left.+\tau_{f}\right)\end{array}$ \\
\hline & $\begin{array}{l}l_{1}(k+1) \\
=l_{2}(k+1)\end{array}$ & $\begin{array}{l}=\max \left(t_{1}(k)\right. \\
\left.+\tau_{g}, t_{3}(k)+\tau_{\Delta}\right) \\
=\max \left(t_{1}(k)\right. \\
\left.+\tau_{g}, t_{4}(k)+\tau_{\Delta}\right)\end{array}$ \\
\hline \multirow{2}{*}{$\begin{array}{l}\{3,4\} \\
<\{1,2\}\end{array}$} & $\begin{array}{l}t_{3}(k+1) \\
=t_{4}(k+1)\end{array}$ & $\begin{array}{l}=\max \left(t_{3}(k)+\tau_{g}\right. \\
+\tau_{f}, t_{1}(k+1) \\
\left.+\tau_{\Delta}+\tau_{f}\right) \\
=\max \left(t_{3}(k)+\tau_{g}\right. \\
+\tau_{f}, t_{2}(k+1) \\
\left.+\tau_{\Delta}+\tau_{f}\right)\end{array}$ \\
\hline & $\begin{array}{l}l_{3}(k+1) \\
=l_{4}(k+1)\end{array}$ & $\begin{array}{l}=\max \left(t_{1}(k)\right. \\
+\tau_{g}, t_{1}(k+1) \\
\left.+\tau_{\Delta}\right) \\
=\max \left(t_{1}(k)\right. \\
+\tau_{g}, t_{2}(k+1) \\
\left.+\tau_{\Delta}\right)\end{array}$ \\
\hline
\end{tabular}


Then, the quadruped robot motion will be modelled with two motors on the three steps of footwork (Matrix $A$ ). To determine matrix $A$, matrix $A_{0}^{*}$ will be determined first by using the MATLAB program [4] and inputting matrix $A_{0}$ according to equation (15) and (16), obtained in Table 4.

Table 4. Matrix $A_{0}^{*}$ of each leg group

\begin{tabular}{|c|c|c|c|c|c|c|c|c|}
\hline$A_{0}^{*}$ & \multicolumn{8}{|c|}{ Matrix } \\
\hline$A_{0\{1,2\}<\{3,4\}}^{*}$ & {$\left[\begin{array}{l}0 \\
\varepsilon \\
2 \\
2 \\
\varepsilon \\
\varepsilon \\
1 \\
1\end{array}\right.$} & $\begin{array}{l}\varepsilon \\
0 \\
2 \\
2 \\
\varepsilon \\
\varepsilon \\
1 \\
1\end{array}$ & $\begin{array}{l}\varepsilon \\
\varepsilon \\
0 \\
\varepsilon \\
\varepsilon \\
\varepsilon \\
\varepsilon \\
\varepsilon\end{array}$ & $\begin{array}{l}\varepsilon \\
\varepsilon \\
\varepsilon \\
0 \\
\varepsilon \\
\varepsilon \\
\varepsilon \\
\varepsilon\end{array}$ & $\begin{array}{l}1 \\
\varepsilon \\
3 \\
3 \\
0 \\
\varepsilon \\
2 \\
2\end{array}$ & $\begin{array}{l}\varepsilon \\
1 \\
3 \\
3 \\
\varepsilon \\
0 \\
2 \\
2\end{array}$ & $\begin{array}{l}\varepsilon \\
\varepsilon \\
1 \\
\varepsilon \\
\varepsilon \\
\varepsilon \\
0 \\
\varepsilon\end{array}$ & $\left.\begin{array}{l}\varepsilon \\
\varepsilon \\
\varepsilon \\
1 \\
\varepsilon \\
\varepsilon \\
\varepsilon \\
0\end{array}\right]$ \\
\hline$A_{0\{1,3\}<\{2,4\}}^{*}$ & {$\left[\begin{array}{l}0 \\
\varepsilon \\
2 \\
2 \\
\varepsilon \\
\varepsilon \\
1 \\
1\end{array}\right.$} & $\begin{array}{l}\varepsilon \\
0 \\
2 \\
2 \\
\varepsilon \\
\varepsilon \\
1 \\
1\end{array}$ & $\begin{array}{l}\varepsilon \\
\varepsilon \\
0 \\
\varepsilon \\
\varepsilon \\
\varepsilon \\
\varepsilon \\
\varepsilon\end{array}$ & $\begin{array}{l}\varepsilon \\
\varepsilon \\
\varepsilon \\
0 \\
\varepsilon \\
\varepsilon \\
\varepsilon \\
\varepsilon\end{array}$ & $\begin{array}{l}1 \\
\varepsilon \\
3 \\
3 \\
0 \\
\varepsilon \\
2 \\
2\end{array}$ & $\begin{array}{l}\varepsilon \\
1 \\
3 \\
3 \\
\varepsilon \\
0 \\
2 \\
2\end{array}$ & $\begin{array}{l}\varepsilon \\
\varepsilon \\
1 \\
\varepsilon \\
\varepsilon \\
\varepsilon \\
0 \\
\varepsilon\end{array}$ & $\left.\begin{array}{l}\varepsilon \\
\varepsilon \\
\varepsilon \\
1 \\
\varepsilon \\
\varepsilon \\
\varepsilon \\
0\end{array}\right]$ \\
\hline$A_{0\{1,4\}<\{2,3\}}^{*}$ & {$\left[\begin{array}{l}0 \\
\varepsilon \\
2 \\
2 \\
\varepsilon \\
\varepsilon \\
1 \\
1\end{array}\right.$} & $\begin{array}{l}\varepsilon \\
0 \\
2 \\
2 \\
\varepsilon \\
\varepsilon \\
1 \\
1\end{array}$ & $\begin{array}{l}\varepsilon \\
\varepsilon \\
0 \\
\varepsilon \\
\varepsilon \\
\varepsilon \\
\varepsilon \\
\varepsilon\end{array}$ & $\begin{array}{l}\varepsilon \\
\varepsilon \\
\varepsilon \\
0 \\
\varepsilon \\
\varepsilon \\
\varepsilon \\
\varepsilon\end{array}$ & $\begin{array}{l}1 \\
\varepsilon \\
3 \\
3 \\
0 \\
\varepsilon \\
2 \\
2\end{array}$ & $\begin{array}{l}\varepsilon \\
1 \\
3 \\
3 \\
\varepsilon \\
0 \\
2 \\
2\end{array}$ & $\begin{array}{l}\mathcal{E} \\
\varepsilon \\
1 \\
\varepsilon \\
\varepsilon \\
\varepsilon \\
0 \\
\varepsilon\end{array}$ & $\left.\begin{array}{l}\varepsilon \\
\varepsilon \\
\varepsilon \\
1 \\
\varepsilon \\
\varepsilon \\
\varepsilon \\
0\end{array}\right]$ \\
\hline
\end{tabular}

After finding the matrix $A_{0}^{*}$, then the matrix $A_{1}$ construction will be searched. To determine the matrix $A_{1}$, we use equation (15) and are obtained in Table 5.

Next, the structure of matrix $A$ based on equation (17) will be determined using the MATLAB Program [4], and it is found in Table 6.
Table 5. Matrix $A_{1}$ of each leg group

\begin{tabular}{|c|c|c|c|c|c|c|c|c|}
\hline$A_{1}$ & \multicolumn{8}{|c|}{ Matrix } \\
\hline \multirow{8}{*}{$A_{1\{1,2\}<\{3,4\}}$} & Г0 & $\varepsilon$ & $\varepsilon$ & $\varepsilon$ & $\mathcal{E}$ & $\varepsilon$ & $\mathcal{E}$ & $\varepsilon]$ \\
\hline & $\mathcal{E}$ & 0 & $\varepsilon$ & $\varepsilon$ & $\mathcal{\varepsilon}$ & $\varepsilon$ & $\mathcal{\varepsilon}$ & $\varepsilon$ \\
\hline & $\mathcal{E}$ & $\varepsilon$ & 0 & $\varepsilon$ & $\varepsilon$ & $\varepsilon$ & $\mathcal{E}$ & $\varepsilon$ \\
\hline & $\mathcal{\varepsilon}$ & $\mathcal{\varepsilon}$ & $\varepsilon$ & 0 & $\varepsilon$ & $\varepsilon$ & $\varepsilon$ & $\varepsilon$ \\
\hline & 2 & $\varepsilon$ & 1 & 1 & 0 & $\varepsilon$ & $\varepsilon$ & $\mathcal{E}$ \\
\hline & $\mathcal{E}$ & 2 & 1 & 1 & $\mathcal{E}$ & 0 & $\varepsilon$ & $\varepsilon$ \\
\hline & $\varepsilon$ & $\varepsilon$ & 2 & $\varepsilon$ & $\varepsilon$ & $\varepsilon$ & 0 & $\varepsilon$ \\
\hline & $L_{\varepsilon}$ & $\varepsilon$ & $\varepsilon$ & 2 & $\mathcal{\varepsilon}$ & $\varepsilon$ & $\mathcal{E}$ & ] \\
\hline \multirow{8}{*}{$A_{1\{1,2\}<\{3,4\}}$} & $\Gamma^{0}$ & $\varepsilon$ & $\varepsilon$ & $\varepsilon$ & $\varepsilon$ & $\varepsilon$ & $\varepsilon$ & $\varepsilon]$ \\
\hline & $\varepsilon$ & 0 & $\varepsilon$ & $\varepsilon$ & $\varepsilon$ & $\varepsilon$ & $\varepsilon$ & $\varepsilon$ \\
\hline & $\varepsilon$ & $\varepsilon$ & 0 & $\varepsilon$ & $\varepsilon$ & $\varepsilon$ & $\varepsilon$ & $\varepsilon$ \\
\hline & $\mathcal{E}$ & $\mathcal{E}$ & $\varepsilon$ & 0 & $\mathcal{\varepsilon}$ & $\varepsilon$ & $\varepsilon$ & $\varepsilon$ \\
\hline & 2 & $\varepsilon$ & 1 & 1 & 0 & $\varepsilon$ & $\varepsilon$ & $\varepsilon$ \\
\hline & $\varepsilon$ & 2 & 1 & 1 & $\mathcal{E}$ & 0 & $\varepsilon$ & $\varepsilon$ \\
\hline & $\varepsilon$ & $\varepsilon$ & 2 & $\varepsilon$ & $\varepsilon$ & $\varepsilon$ & 0 & $\varepsilon$ \\
\hline & $-\varepsilon$ & $\varepsilon$ & $\varepsilon$ & 2 & $\varepsilon$ & $\varepsilon$ & $\varepsilon$ & لـ \\
\hline \multirow{8}{*}{$A_{1\{1,2\}<\{3,4\}}$} & Г0 & $\mathcal{E}$ & $\varepsilon$ & $\mathcal{E}$ & $\mathcal{E}$ & $\varepsilon$ & $\varepsilon$ & $\varepsilon\rceil$ \\
\hline & $\varepsilon$ & 0 & $\varepsilon$ & $\varepsilon$ & $\varepsilon$ & $\varepsilon$ & $\mathcal{E}$ & $\varepsilon$ \\
\hline & $\varepsilon$ & $\varepsilon$ & 0 & $\varepsilon$ & $\varepsilon$ & $\varepsilon$ & $\varepsilon$ & $\varepsilon$ \\
\hline & $\varepsilon$ & $\varepsilon$ & $\varepsilon$ & 0 & $\varepsilon$ & $\varepsilon$ & $\varepsilon$ & $\varepsilon$ \\
\hline & 2 & $\varepsilon$ & 1 & 1 & 0 & $\varepsilon$ & $\varepsilon$ & $\varepsilon$ \\
\hline & $\varepsilon$ & 2 & 1 & 1 & $\varepsilon$ & 0 & $\mathcal{E}$ & $\varepsilon$ \\
\hline & $\varepsilon$ & $\varepsilon$ & 2 & $\varepsilon$ & $\varepsilon$ & $\varepsilon$ & 0 & $\varepsilon$ \\
\hline & $L_{\varepsilon}$ & $\varepsilon$ & $\varepsilon$ & 2 & $\varepsilon$ & $\varepsilon$ & $\varepsilon$ & لـ \\
\hline
\end{tabular}

A matrix of all is modelling the quadruped robot motion with two motors and the leg already defined. Matrix A is also used to determine the periodization of the motion steps of each group of legs adjusted to their respective eigenvectors. A is a matrix system constructed from equation (11) which contains a combination of $t_{i}(k)$ and $l_{i}(k)$, where $t_{i}(k)$ is the time when foot $i$ touch down at iteration $k$ and $l_{i}(k)$ is the time when foot $i$ lift off at iteration $k$. Because $i=1,2,3,4$ then the size of matrix A is $8 \times 8$. 
Table 6. Matrix structure of each leg group

\begin{tabular}{|c|c|c|c|c|c|c|c|c|}
\hline$A_{1}$ & \multicolumn{8}{|c|}{ Matrix } \\
\hline$A_{\{1,2\}<\{3,4\}}$ & {$\left[\begin{array}{l}0 \\
3 \\
5 \\
5 \\
\varepsilon \\
2 \\
4 \\
4\end{array}\right.$} & $\begin{array}{l}\varepsilon \\
3 \\
5 \\
5 \\
\varepsilon \\
2 \\
4 \\
4\end{array}$ & $\begin{array}{l}2 \\
2 \\
4 \\
4 \\
1 \\
1 \\
3 \\
3\end{array}$ & $\begin{array}{l}2 \\
2 \\
4 \\
4 \\
1 \\
1 \\
3 \\
3\end{array}$ & $\begin{array}{l}1 \\
\varepsilon \\
3 \\
3 \\
0 \\
\varepsilon \\
2 \\
2\end{array}$ & $\begin{array}{l}\varepsilon \\
1 \\
3 \\
3 \\
\varepsilon \\
\varepsilon \\
2 \\
2\end{array}$ & $\begin{array}{l}\varepsilon \\
\varepsilon \\
1 \\
\varepsilon \\
\varepsilon \\
\varepsilon \\
0 \\
\varepsilon\end{array}$ & $\left.\begin{array}{l}\varepsilon \\
\varepsilon \\
\varepsilon \\
1 \\
\varepsilon \\
\varepsilon \\
\varepsilon \\
0\end{array}\right]$ \\
\hline$A_{\{1,2\}<\{3,4\}}$ & {$\left[\begin{array}{l}0 \\
3 \\
5 \\
5 \\
\varepsilon \\
2 \\
4 \\
4\end{array}\right.$} & $\begin{array}{l}\varepsilon \\
3 \\
5 \\
5 \\
\varepsilon \\
2 \\
4 \\
4\end{array}$ & $\begin{array}{l}2 \\
2 \\
4 \\
4 \\
1 \\
1 \\
3 \\
3\end{array}$ & $\begin{array}{l}2 \\
2 \\
4 \\
4 \\
1 \\
1 \\
3 \\
3\end{array}$ & $\begin{array}{l}1 \\
\varepsilon \\
3 \\
3 \\
0 \\
\varepsilon \\
2 \\
2\end{array}$ & $\begin{array}{l}\varepsilon \\
1 \\
3 \\
3 \\
\varepsilon \\
\varepsilon \\
2 \\
2\end{array}$ & $\begin{array}{l}\varepsilon \\
\varepsilon \\
1 \\
\varepsilon \\
\varepsilon \\
\varepsilon \\
0 \\
\varepsilon\end{array}$ & $\left.\begin{array}{l}\varepsilon \\
\varepsilon \\
\varepsilon \\
1 \\
\varepsilon \\
\varepsilon \\
\varepsilon \\
0\end{array}\right]$ \\
\hline$A_{\{1,2\}<\{3,4\}}$ & {$\left[\begin{array}{l}0 \\
3 \\
5 \\
5 \\
\varepsilon \\
2 \\
4 \\
4\end{array}\right.$} & $\begin{array}{l}\varepsilon \\
3 \\
5 \\
5 \\
\varepsilon \\
2 \\
4 \\
4\end{array}$ & $\begin{array}{l}2 \\
2 \\
4 \\
4 \\
1 \\
1 \\
3 \\
3\end{array}$ & $\begin{array}{l}2 \\
2 \\
4 \\
4 \\
1 \\
1 \\
3 \\
3\end{array}$ & $\begin{array}{l}1 \\
\varepsilon \\
3 \\
3 \\
0 \\
\varepsilon \\
2 \\
2\end{array}$ & $\begin{array}{l}\varepsilon \\
1 \\
3 \\
3 \\
\varepsilon \\
\varepsilon \\
2 \\
2\end{array}$ & $\begin{array}{l}\varepsilon \\
\varepsilon \\
1 \\
\varepsilon \\
\varepsilon \\
\varepsilon \\
0 \\
\varepsilon\end{array}$ & $\left.\begin{array}{l}\varepsilon \\
\varepsilon \\
\varepsilon \\
1 \\
\varepsilon \\
\varepsilon \\
\varepsilon \\
0\end{array}\right]$ \\
\hline
\end{tabular}

\subsection{Analysis of Quadruped Robot Motion with Two Motors}

After modelling the quadruped robot motion, the periodic system of each motion will be discussed next. By using the Scilab Program [7] because the matrix $A \in \mathbb{R}_{\max }^{n \times n}$ is irreducible, then according to Theorem 3 , the matrix $A$ has a single Max-Plus algebra eigenvalue for each leg group $(\{1,2\} \prec$ $\{3,4\},\{1,3\} \prec\{2,4\},\{1,4\} \prec\{2,3\})$ i.e. $\lambda=4$. Because $\lambda \in \mathbb{R}$ is the eigenvalue of the Max-Plus matrix $\mathrm{A}$, then according to Theorem $2, \lambda=4$ is the average weight of a circuit in $G(A)$. Next, will determined the eigenvectors from each of the following groups of legs.

Table 7. Eigenvectors of Each Leg Group

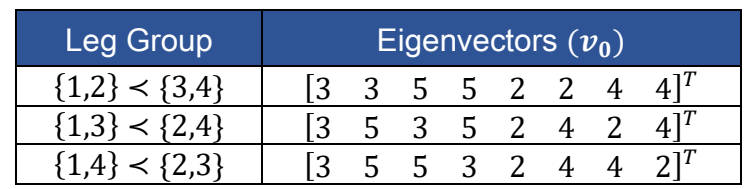

Based on Table 7, it is known that the eigenvectors for all groups of robot legs are the same size, which is $8 \times 1$. The first four rows represent the time of the leg $i$ touch down $\left(t_{i}\right)$, where $i=1, \ldots, 4$ and rows 5 to 8 represent the time of the leg $i$ lift off $\left(l_{i}\right)$ for $i=5, \ldots, 8$. Based on this table it is also known that the eigenvectors for all groups of legs in the robot are different from each other. This can be seen in the different eigenvector matrix structures.
This difference is caused by the elements in the eigenvectors produced in accordance with the order of the movement of the legs in each group of robot legs. For example eigenvectors $\left[\begin{array}{llllllll}3 & 3 & 5 & 5 & 2 & 2 & 4 & 4\end{array}\right]^{T}$ represents the lift off and touch down time of each foot in the foot group $\{1,2\} \prec\{3,4\}$. Note that the first and second rows have the same value, that is 3 , and the fifth and sixth rows are equal to 2 . This shows that when $t=$ 3 , legs 1 and 2 touch down and then when $t=2$, legs 1 and 2 lift off. Furthermore, as the initial time runs, then there must be at least a vector component with a zero value or in other words each vector is reduced by the smallest vector component, i.e 2 so that it becomes:

$$
\boldsymbol{v}_{0}^{*}=\left[\begin{array}{llllllll}
1 & 1 & 3 & 3 & 0 & 0 & 2 & 2
\end{array}\right]^{T} \text {. The same }
$$
explanation also applies to feet 3 and 4 .

Quadruped robot has two motors with the speed of both motors and the time of the two motors to move the legs of the robot are the same. From this, the initial position of the robot to move cannot be in a perfect standing position (the robot does not stand up straight). If in a state of perfect standing, then the legs of the robot will step simultaneously so that the robot moves like dragging. Therefore, the positions of the feet are arranged so that the robot can move periodically. The eigenvector signifies that the foot starts periodic movements in an iteration. This can be seen in Table 8 and Table 9. The following two tables will show the periodic iteration of each leg group:

Table 8. Periodic Iteration all-of the legs with $x(0)=0$

\begin{tabular}{|c|c|c|c|c|c|c|c|c|c|}
\hline \multirow{2}{*}{\begin{tabular}{|l} 
Leg \\
Group
\end{tabular}} & \multicolumn{9}{|c|}{ Periodic Iteration } \\
\hline & $k$ & $t_{1}$ & $t_{2}$ & $t_{3}$ & $t_{4}$ & $l_{1}$ & $l_{2}$ & $l_{3}$ & $l_{4}$ \\
\hline \multirow{6}{*}{$\begin{array}{l}\{1,2\}< \\
\{3,4\}\end{array}$} & 0 & 0 & 0 & 0 & 0 & 0 & 0 & 0 & 0 \\
\hline & 1 & 3 & 3 & 5 & 5 & 2 & 2 & 4 & 4 \\
\hline & 2 & 7 & 7 & 9 & 9 & 6 & 6 & 8 & 8 \\
\hline & 3 & 11 & 11 & 13 & 13 & 10 & 10 & 12 & 12 \\
\hline & 4 & 15 & 15 & 17 & 17 & 14 & 14 & 16 & 16 \\
\hline & $\vdots$ & $\vdots$ & 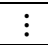 & $\vdots$ & $\vdots$ & $\vdots$ & $\vdots$ & $\vdots$ & $\vdots$ \\
\hline \multirow{6}{*}{$\begin{array}{l}\{1,3\}< \\
\{2,4\}\end{array}$} & 0 & 0 & U & 0 & 0 & 0 & 0 & 0 & 0 \\
\hline & 1 & 3 & 5 & 3 & 5 & 2 & 4 & 2 & 4 \\
\hline & 2 & 7 & 9 & 7 & 9 & 6 & 8 & 6 & 8 \\
\hline & 3 & 11 & 13 & 11 & 13 & 10 & 12 & 10 & 12 \\
\hline & 4 & 15 & 17 & 15 & 17 & 14 & 16 & 14 & 16 \\
\hline & $\vdots$ & $\vdots$ & $\vdots$ & $\vdots$ & $\vdots$ & $\vdots$ & $\vdots$ & $\vdots$ & $\vdots$ \\
\hline \multirow{6}{*}{$\begin{array}{c}\{1,4\} \prec \\
\{2,3\}\end{array}$} & 0 & 0 & 0 & 0 & 0 & 0 & 0 & 0 & 0 \\
\hline & 1 & 3 & 5 & 5 & 3 & 2 & 4 & 4 & 2 \\
\hline & 2 & 7 & 9 & 9 & 7 & 6 & 8 & 8 & 6 \\
\hline & 3 & 11 & 13 & 13 & 11 & 10 & 12 & 12 & 10 \\
\hline & 4 & 15 & 17 & 17 & 15 & 14 & 16 & 16 & 14 \\
\hline & $\vdots$ & $\vdots$ & $\vdots$ & $\vdots$ & $\vdots$ & $\vdots$ & $\vdots$ & $\vdots$ & $\vdots$ \\
\hline
\end{tabular}


Table 9. Periodic Iteration all of the legs with $x(0)=v_{0}^{*}$

\begin{tabular}{|c|c|c|c|c|c|c|c|c|c|}
\hline \multirow{2}{*}{$\begin{array}{l}\text { Leg } \\
\text { Group }\end{array}$} & \multicolumn{9}{|c|}{ Periodic Iteration } \\
\hline & $k$ & $t_{1}$ & $t_{2}$ & $t_{3}$ & $t_{4}$ & $l_{1}$ & $l_{2}$ & $l_{3}$ & $l_{4}$ \\
\hline \multirow{6}{*}{$\begin{array}{l}\{1,2\}< \\
\{3,4\}\end{array}$} & 0 & 1 & 1 & 3 & 3 & 0 & 0 & 2 & 2 \\
\hline & 1 & 5 & 5 & 7 & 7 & 4 & 4 & 6 & 6 \\
\hline & 2 & y & 9 & 11 & 11 & 8 & 8 & 10 & 10 \\
\hline & 3 & 13 & 13 & 15 & 15 & 12 & 12 & 14 & 14 \\
\hline & 4 & 17 & 17 & 19 & 19 & 16 & 16 & 18 & 18 \\
\hline & $\vdots$ & & $\vdots$ & $\vdots$ & $\vdots$ & $\vdots$ & $\vdots$ & $\vdots$ & $\vdots$ \\
\hline \multirow{6}{*}{$\begin{array}{l}\{1,3\}< \\
\{2,4\}\end{array}$} & & & 3 & 1 & 3 & 0 & 2 & 0 & 2 \\
\hline & 1 & 5 & 7 & 5 & 7 & 4 & 6 & 4 & 6 \\
\hline & 2 & 9 & 11 & 9 & 11 & 8 & 10 & 8 & 10 \\
\hline & 3 & 13 & 15 & 13 & 15 & 12 & 14 & 12 & 14 \\
\hline & 4 & 17 & 19 & 17 & 19 & 16 & 18 & 16 & 18 \\
\hline & $\vdots$ & & $\%$ & $\vdots$ & $\vdots$ & $\vdots$ & $\vdots$ & $\vdots$ & $\vdots$ \\
\hline \multirow{6}{*}{$\begin{array}{c}\{1,4\}< \\
\{2,3\}\end{array}$} & 0 & & 3 & 3 & 1 & 0 & 2 & 2 & 0 \\
\hline & 1 & 4 & 7 & 7 & 5 & 4 & 6 & 6 & 4 \\
\hline & 2 & 9 & 11 & 11 & 9 & 8 & 10 & 10 & 8 \\
\hline & 3 & 13 & 15 & 15 & 13 & 12 & 14 & 14 & 12 \\
\hline & 4 & 17 & 19 & 19 & 17 & 16 & 18 & 18 & 16 \\
\hline & $\vdots$ & $\vdots$ & $\vdots$ & $\vdots$ & $\vdots$ & $\vdots$ & $\vdots$ & $\vdots$ & $\vdots$ \\
\hline
\end{tabular}

Based on Table 8, it is known that initially all robot legs are in the same condition namely touch down or $x(0)=\left[\begin{array}{llllllll}0 & 0 & 0 & 0 & 0 & 0 & 0 & 0\end{array}\right]^{T}$. Next, suppose we take one of the conditions in the foot group $\{1,2\} \prec\{3,4\}$, from Table 8 we can observe that leg 1 and 2 begin to lift off at $t=2$ and begin to touch down again at $=3$. Furthermore, when $t=4$, feet 3 and 4 lift off and touch down back when $t=5$. It is clear that it takes time $x(0)=$ $\left[\begin{array}{llllllll}0 & 0 & 0 & 0 & 0 & 0 & 0 & 0\end{array}\right]^{T}$ for each leg to touch down simultaneously and this corresponds to the value of the parameter $\tau_{\Delta}$ used. In the first iteration the time needed for each leg starting from a stationary position is different, where as in the second iteration, the time of lift off and touch down required by each leg increases according to the eigenvalue $\lambda=4$. Note, in the second iteration, the time required for each leg to make movements when lift off and touch down is the same as the eigenvector structure. After the second iteration the movement of all legs starts periodically, increasing by $\lambda=4$ for the next iteration.

Look at Table 9, according to Definition 4 and $\boldsymbol{v}_{\mathbf{0}}^{*}$ is a linear combination max-plus of $\boldsymbol{v}_{\mathbf{0}}$, then vector $\boldsymbol{v}^{*}$ is an eigenvector corresponding to $\lambda_{\max }(A)$. The iteration process of the latest eigenvector is the same as shown in Table 9, where in it is known that in the 0th iteration the time used is the same as the eigenvector then for the next iteration all legs move periodically. The condition of the other leg groups is also the same as the leg group $\{1,2\} \prec\{3,4\}$, where in the second iteration the time required by each leg increases with the magnitude corresponding to the eigenvalue, so for subsequent iterations each leg starts periodically.

\section{CONCLUSION}

Robot Lego Mainstroms EV3 four-legged robot consists of several main constituent components such as construction of the robot body, EV3 Brick (controlling robot), two motors (moving the robot's legs) and connectors (connecting EV3 Brick with the motor). There are three groups of legs when walking namely $\{1,2\} \prec\{3,4\},\{1,4\} \prec\{2,3\}$ and $\{1,3\} \prec$ $\{2,4\}$. The movement of a quadruped robot with two motors means that two legs that are in the same group do the same movements both when floating and stepping and alternating with other groups of legs. Then to model when all the legs move in an iteration, just model one of the legs in each group of legs as in Table 3 . When modelling motion robot, a matrix of $8 \times 8$ is needed. From this matrix, it can be seen the total time needed for a leg to make a movement in one cycle (lift off then touch down) by looking at the eigenvalue. The eigenvalues obtained for all foot groups are the same. In addition, from this matrix, we can find out when each leg starts to make periodic movements, which is when the time needed for each leg to do floating and tread movements is the same as the elements in the eigenvector

\section{ACKNOWLEDGMENTS}

We would like to thank the Sanata Dharma University Research Institute for providing support and research funding in Magister Research Field

\section{REFERENCES}

[1] H. Herwin, K. Harianto, and C.K. Wibawa, Robot Auto Pointing Sebagai Media Pencarian Sinyal Terbaik Pada Instalasi Radio Wireless Menggunakan Mikrokontroler Arduino Uno, INOVTEK Polbeng - Seri Inform, 2019.

[2] A. Kausar and V.N. Sari, J, Robot Pencari Alamat Menggunakan Warna, Media Infotama, 2014.

[3] A. Hidayat, Design and Implementation Inverse Kinematics and Sine Pattern Methods for Locomotion Control on Autonomous Quadruped Robot, in EProceeding Eng. (Bandung, 2016), p. 2116. 
[4] M.A. Rudhito, Aljabar Max-Plus Dan Penerapannya (Universitas Sanata Dharma, Yogyakarta, 2016).

[5] B. Kersbergen, G.A.D. Lopes, T.J.J. Van Den Boom, B. De Schutter, and R. Babuška, Optimal gait switching for legged locomotion in IEEE Int. Conf. Intell. Robot. Syst. 2011.

[6] K. Kozłowski, M. Tokhi, and G. Virk, Mobile Service Robotics (World Scientific, Singapore, 2014).

[7] Subiono, Aljabar Min-Max Plus Dan Terapannya (Institut Teknologi Sepuluh November, Surabaya, 2015). 